\title{
Computational Fluid Dynamics Analysis of Balloon-Expandable Coronary Stents: Influence of Stent and Vessel Deformation
}

\author{
David Martin \\ Technological University Dublin, david.martin@tudublin.ie \\ Eoin Murphy \\ Technological University Dublin, eoin.murphy@tudublin.ie \\ Fergal Boyle \\ Technological University Dublin, fergal.boyle@tudublin.ie
}

Follow this and additional works at: https://arrow.tudublin.ie/engschmecart

\section{Recommended Citation}

Martin DM, et al. Computational fluid dynamics analysis of balloon-expandable coronary stents: Influence of stent and vessel deformation. Med Eng Phys (2014), doi:10.1016/j.medengphy.2014.05.011

This Article is brought to you for free and open access by the School of Mechanical and Design Engineering at ARROW@TU Dublin. It has been accepted for inclusion in Articles by an authorized administrator of ARROW@TU Dublin. For more information, please contact arrow.admin@tudublin.ie, aisling.coyne@tudublin.ie, gerard.connolly@tudublin.ie.

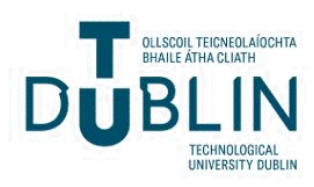




\title{
Computational fluid dynamics analysis of balloon-expandable coronary stents: Influence of stent and vessel deformation
}

\author{
David M. Martin, Eoin A. Murphy, Fergal J. Boyle* \\ Department of Mechanical Engineering, Dublin Institute of Technology, Bolton Street, Dublin 1, Ireland
}

\section{A R T I C L E I N F O}

\section{Article history:}

Received 20 August 2013

Received in revised form 7 May 2014

Accepted 13 May 2014

\section{Keywords:}

Coronary heart disease

Coronary stent

Restenosis

Computational fluid dynamics

Wall shear stress

Wall shear stress gradient

Oscillatory shear index

\begin{abstract}
A B S T R A C T
In many computational fluid dynamics (CFD) studies of stented vessel haemodynamics, the geometry of the stented vessel is described using non-deformed (NDF) geometrical models. These NDF models neglect complex physical features, such as stent and vessel deformation, which may have a major impact on the haemodynamic environment in stented coronary arteries. In this study, CFD analyses were carried out to simulate pulsatile flow conditions in both NDF and realistically-deformed (RDF) models of three stented coronary arteries. While the NDF models were completely idealised, the RDF models were obtained from nonlinear structural analyses and accounted for both stent and vessel deformation. Following the completion of the CFD analyses, major differences were observed in the time-averaged wall shear stress (TAWSS), time-averaged wall shear stress gradient (TAWSSG) and oscillatory shear index (OSI) distributions predicted on the luminal surface of the artery for the NDF and RDF models. Specifically, the inclusion of stent and vessel deformation in the CFD analyses resulted in a 32\%, 30\% and 31\% increase in the area-weighted mean TAWSS, a 3\%, 7\% and 16\% increase in the area-weighted mean TAWSSG and a $21 \%, 13 \%$ and $21 \%$ decrease in the area-weighted mean OSI for Stents A, B and C, respectively. These results suggest that stent and vessel deformation are likely to have a major impact on the haemodynamic environment in stented coronary arteries. In light of this observation, it is recommended that these features are considered in future CFD studies of stented vessel haemodynamics.
\end{abstract}

(C) 2014 IPEM. Published by Elsevier Ltd. All rights reserved.

\section{Introduction}

In recent years several clinical studies have identified a strong correlation between altered vessel haemodynamics and neointimal hyperplasia following coronary stent deployment [1-4]. In light of this observation a significant body of research has been carried out to investigate the relationship between coronary stent design and altered vessel haemodynamics. Due to the difficulty involved in the evaluation of the haemodynamic impact of stent deployment in an in vivo setting, a large portion of this research has been carried out using computational fluid dynamics (CFD) [5-33]. In the vast majority of these studies, however, the geometry of both the stent and the lumen is described using idealised non-deformed (NDF) geometrical models. These idealised NDF models neglect a number of complex features, such as stent and vessel deformation, which may have a major impact on the haemodynamic environment in a stented coronary artery. Balloon-expandable coronary stents, for example, expand in a highly non-uniform manner and experience both longitudinal foreshortening and radial recoil during their

\footnotetext{
* Corresponding author. Tel.: +3531402 3813 .

E-mail address: fergal.boyle@dit.ie (F.J. Boyle).
}

deployment. The artery also deforms around the expanded stent causing prolapsed tissue at the stent extremities and between neighbouring struts. Although these features may have a major impact on the haemodynamic environment in stented coronary arteries, they are routinely neglected in CFD studies of stented vessel haemodynamics.

In light of this observation, the aim of this study was to determine whether stent and vessel deformation have a major impact on the haemodynamic environment in stented coronary arteries. To investigate the haemodynamic impact of stent and vessel deformation, NDF and realistically-deformed (RDF) models of three stented coronary arteries were first generated. While the NDF models were completely idealised, the RDF models were obtained from nonlinear structural analyses and accounted for both stent and vessel deformation. Transient CFD analyses were then carried out to simulate pulsatile flow conditions in each of the NDF and RDF models of the stented coronary arteries. Following the completion of the analyses, the haemodynamic environment predicted in each of the NDF and RDF models was evaluated in terms of the time-averaged wall shear stress (TAWSS), time-averaged wall shear stress gradient (TAWSSG) and oscillatory shear index (OSI) distributions predicted on the luminal surface of the arteries. To fully-elucidate the haemodynamic impact of stent and vessel deformation, the 
Table 1

Geometrical properties of the investigated stents.

\begin{tabular}{llll}
\hline Stent & A & B & C \\
\hline Stent configuration & Closed-cell & Open-cell & Open-cell \\
Strut thickness & $0.14 \mathrm{~mm}$ & $0.10 \mathrm{~mm}$ & $0.13 \mathrm{~mm}$ \\
Strut width & $0.13 \mathrm{~mm}$ & $0.10 \mathrm{~mm}$ & $0.10 \mathrm{~mm}$ \\
Link configuration & Peak-to-peak & Peak-to-peak & Peak-to-valley \\
Link type & Flexible & Non-flexible & Non-flexible \\
Link shape & N-shaped & Straight & Straight \\
Link width & $0.10 \mathrm{~mm}$ & $0.08 \mathrm{~mm}$ & $0.10 \mathrm{~mm}$ \\
\hline
\end{tabular}

area-weighted mean, standard deviation and skewness of the TAWSS, TAWSSG and OSI distributions were then calculated. Based on the results of the CFD analyses, an optimum strategy for modelling the geometry of the stented coronary lumen in future CFD studies of stented vessel haemodynamics is recommended.

\section{Methodology}

\subsection{Geometry and discretisation}

The three investigated stents are generic stents and are referred to herein as Stent A, Stent B and Stent C. Stent A consists of sinusoidal strut sections that are connected by flexible $\mathrm{N}$-shaped link elements in a closed-cell, peak-to-peak configuration. Stent B consists of modular strut sections which are connected by inflexible straight link elements in an open-cell, peak-to-peak configuration. Finally, Stent $C$ consists of sinusoidal strut sections that are connected by straight link elements in an open-cell, peak-to-valley configuration. Stents $\mathrm{A}, \mathrm{B}$ and $\mathrm{C}$ were assigned a length of $8 \mathrm{~mm}$ and a strut thickness of $0.14,0.10$ and $0.13 \mathrm{~mm}$, respectively. The geometrical properties of each stent are given in Table 1.

In order to generate the NDF models of the stented coronary arteries, NDF models of the expanded stents were first generated in Rhinoceros 3D (McNeel, Indianapolis, IN, USA). These NDF models neglected stent deformation and were assigned a fixed internal diameter of $3 \mathrm{~mm}$. The geometry of the NDF stents was then extracted from that of a uniform cylinder whose outer diameter corresponded to that of the investigated stent. Tapered sections, which measured $1 \mathrm{~mm}$ in length, were then added to the ends of the NDF models to account for a stent-to-artery deployment ratio of $1.1: 1$. Finally, to ensure that the applied boundary conditions had no impact on the flow in the region of interest, an additional entrance and exit length of $32 \mathrm{~mm}$ was added to the ends of the NDF models. The subsequent NDF models were then imported into ANSYS ICEM (ANSYS Inc., Canonsburg, PA, USA) and discretised into tetrahedral/prism meshes using approximately 1.9 million elements. In order to identify a suitable mesh density for the NDF models, a mesh convergence study was carried out and the results of this study are presented in Appendix A. The procedure adopted to generate the NDF models is demonstrated in Fig. 1 while the NDF models of each of the investigated stents are shown in Fig. 2.

To generate the corresponding RDF models of the stented coronary arteries, nonlinear structural analyses were first carried out using ABAQUS (Dassault Systèmes, Providence, RI, USA) to simulate the deployment of the investigated stents in an idealised cylindrical model of a coronary artery. To this end, geometrical models of the crimped stents were first generated in Rhinoceros 3D and assigned a fixed internal diameter of $1 \mathrm{~mm}$. The crimped stent models were each discretised using approximately 25,000 continuum elements. The artery was then modelled as a uniform straight cylinder and was assigned a length of $20 \mathrm{~mm}$ and internal and external diameters of 2.7 and $4.5 \mathrm{~mm}$, respectively. The artery wall, which had a subsequent thickness of $0.9 \mathrm{~mm}$, was divided into three layers that represented the intima, the media and the adventitia. The intima, media and adventitia were assigned thicknesses of 0.24 , (a)

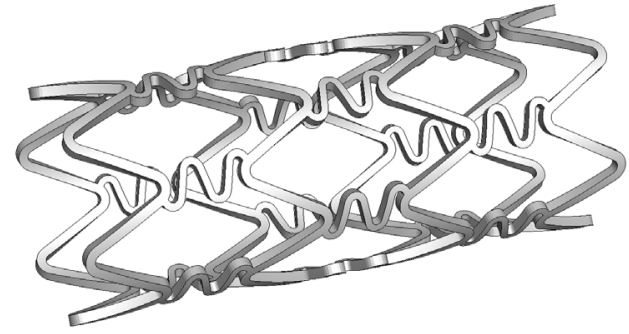

(b)

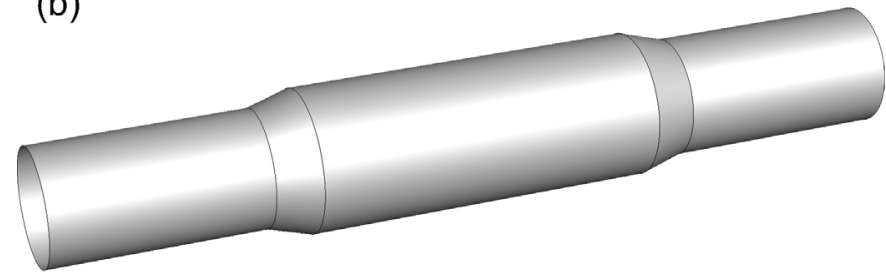

(c)

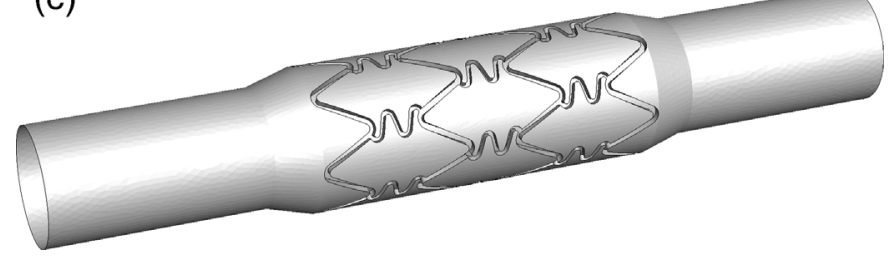

Fig. 1. Procedure employed to extract (a) the NDF models of the investigated stents from, (b) the NDF models of the coronary arteries to generate (c) the NDF models of the stented coronary arteries.

0.32 and $0.34 \mathrm{~mm}$, respectively, and the artery was then discretised using 70,272 continuum elements. To simulate the deployment of the stents, a realistic model of a tri-folded balloon-tipped catheter was generated. The balloon-tipped catheter consisted of a guide wire, a catheter shaft and a folded angioplasty balloon and its configuration has been discussed in a previous study [34]. The guide wire and catheter shaft were discretised using 740 and 13,104 continuum elements, respectively, while the angioplasty balloon was discretised using 12,462 membrane elements. In order to

(a)
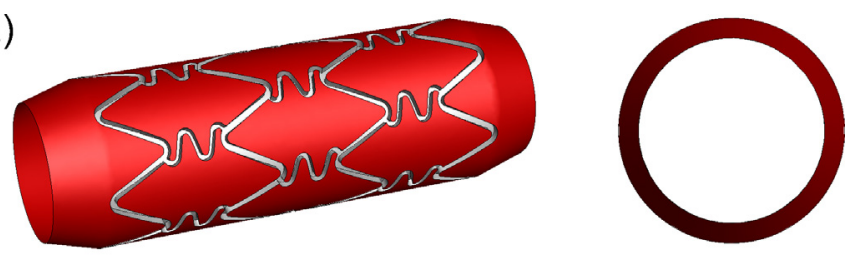

(b)
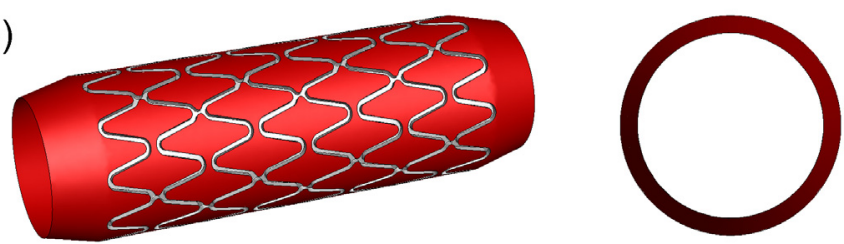

(c)
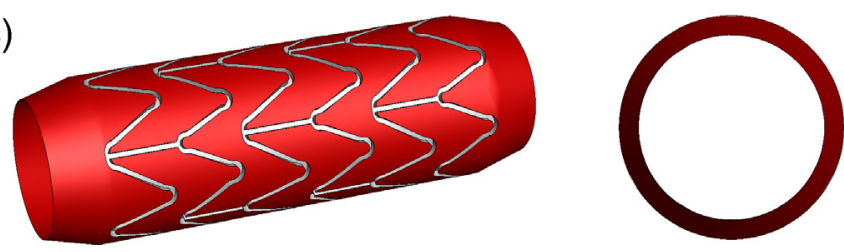

Fig. 2. Isometric (left) and cross-sectional (right) views of the NDF model of (a) Stent A, (b) Stent B and (c) Stent C. 

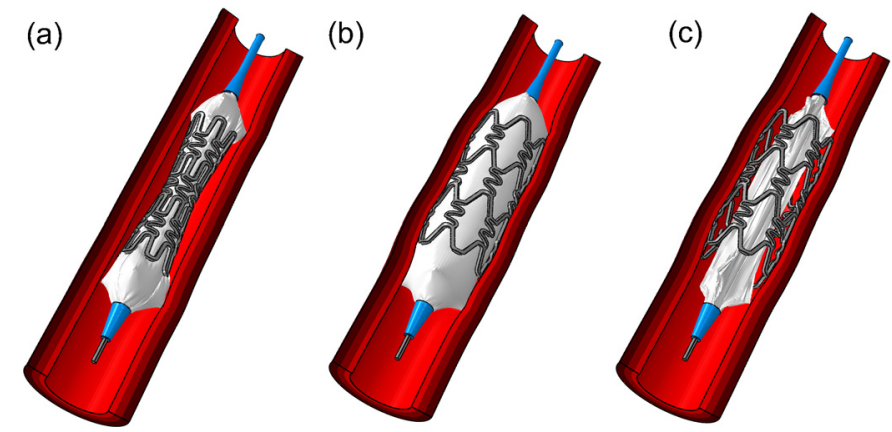

Fig. 3. Deformed configuration of Stent A (a) during loading,(b) at maximum loading and (c) following the unloading of the angioplasty balloon.

identify an appropriate mesh density for the components in the structural analyses, a mesh convergence study was first carried out and the results of this study are presented in Appendix A.

The mechanical behaviour of the three investigated stents was described using a rate-independent elastic-plastic material model with isotropic hardening. The investigated stents were each assumed to be manufactured from $316 \mathrm{~L}$ stainless steel and the data reported by Murphy et al. was employed to describe their elastic-plastic response [35]. The mechanical behaviour of the guide wire, the catheter shaft and the angioplasty balloon was then described using isotropic linear elastic material models, and the data reported by Mortier et al. was used to describe their linear elastic response [36]. Finally, the mechanical behaviour of the intima, the media and the adventitia was described using isotropic hyperelastic material models, and the data reported by Holzapfel et al. was adopted to describe their nonlinear elastic response [37]. In order to simulate the deployment of the investigated stents, a uniform pressure load was applied to the inner surface of the angioplasty balloon. This pressure load was increased until a stent-to-artery deployment ratio of 1.1:1 was achieved and then decreased to a value of $-0.01 \mathrm{MPa}$ to allow both the stent and artery to recoil. To prevent rigid-body motions during the structural analyses, the nodes located at the extremities of the artery and the guide wire were fully constrained while a number of nodes located at the midsection of the stents were constrained in both the circumferential and longitudinal directions.

As the deployment of the stents was highly nonlinear, the ABAQUS/Explicit solver was employed to carry out the structural analyses. As inertia is assumed to have a negligible role during stent deployment, the structural analyses were each carried out using a quasi-static approach. To demonstrate the deployment procedure, the configuration of Stent A is shown during the inflation/deflation of the angioplasty balloon in Fig. 3. Following the completion of the structural analyses, the geometry of the stent and the coronary artery was imported into ANSYS ICEM as a triangulated surface mesh. The distance both proximal and distal to the stent at which the lumen returned to its original diameter was then measured and any elements located beyond these positions were removed. The meshes were then converted into facetted geometries and used to generate the RDF models of the stented coronary arteries. As before, an additional length of $32 \mathrm{~mm}$ was added to the ends of the RDF models to ensure that the applied boundary conditions had no impact on the flow in the region of interest. The RDF models were then discretised into tetrahedral/prism meshes using almost 1.9 million elements. To identify an appropriate mesh density for the RDF models, a mesh convergence study was carried out and the results of this study are presented in Appendix A. The procedure adopted to generate the RDF models is demonstrated in Fig. 4 and the RDF models of each of the investigated stents are shown in Fig. 5. (a)
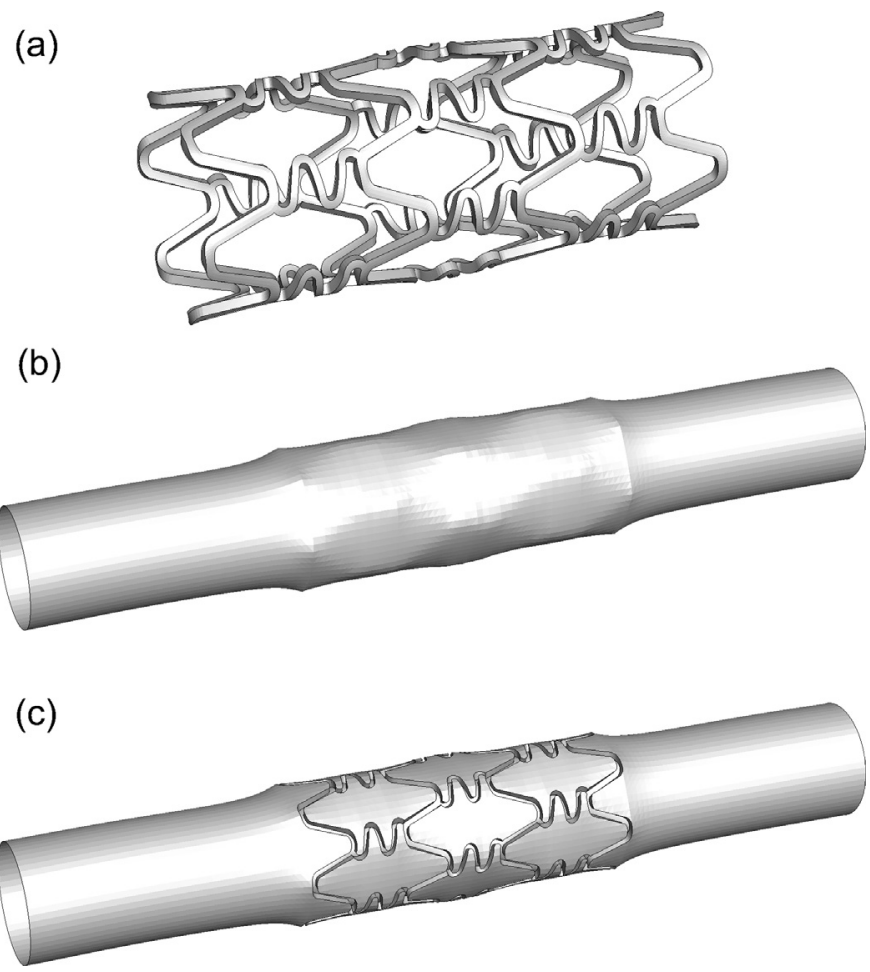

Fig. 4. Procedure employed to extract (a) the RDF models of the investigated stents from, (b) the RDF models of the coronary arteries to generate (c) the RDF models of the stented coronary arteries.

\subsection{Constitutive behaviour}

In order to describe the mechanical behaviour of whole human blood, the mass density of the fluid was assigned a fixed value of $1,050 \mathrm{~kg} / \mathrm{m}^{3}$. As whole human blood exhibits significant shear thinning behaviour at shear rates below $100 \mathrm{~s}^{-1}$, the dynamic viscosity of the fluid was described using the non-Newtonian Bird-Carreau model as follows:

$$
\mu=\mu_{\infty}+\left(\mu_{0}-\mu_{\infty}\right)\left[1+\left(\dot{\gamma} \lambda_{t}\right)^{2}\right]^{((q-1) / 2)}
$$

(a)
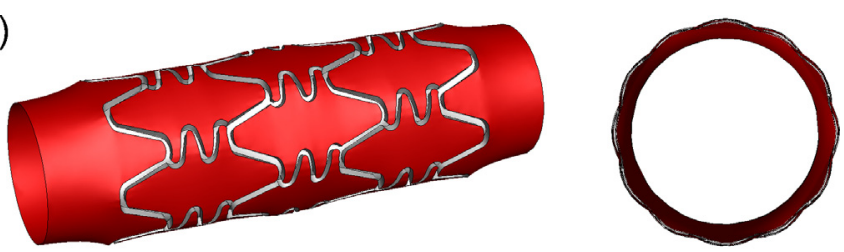

(b)
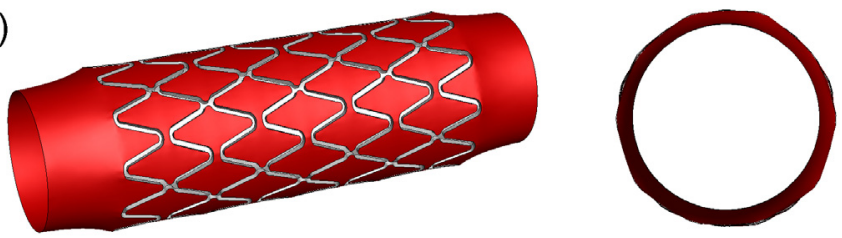

(c)
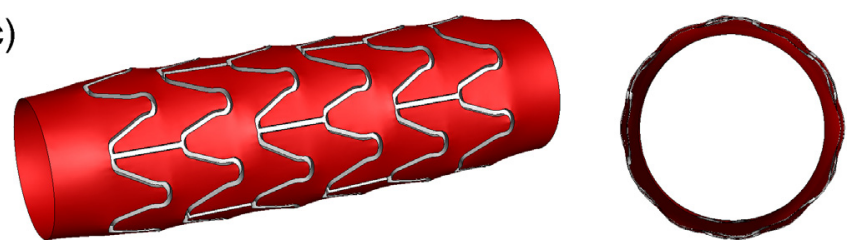

Fig. 5. Isometric (left) and cross-sectional (right) views of the RDF model of (a) Stent A, (b) Stent B and (c) Stent C. 


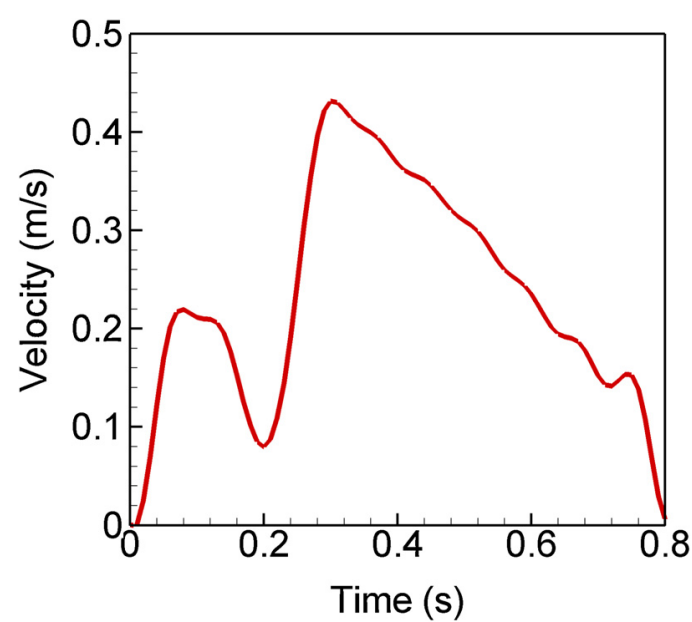

Fig. 6. Transient velocity profile employed to simulate pulsatile flow conditions in a human left-anterior descending coronary artery.

The term $\mu$ denotes the dynamic viscosity, $\mu_{0}$ denotes the low shear viscosity, $\mu_{\infty}$ denotes the high shear viscosity, $\lambda_{t}$ denotes the time constant, $\dot{\gamma}$ denotes the shear rate and $n$ denotes the power law index. The experimental data used to describe the viscous behaviour of the fluid was adopted from Jung et al. who measured the viscosity of whole human blood at a range of shear rates [38]. Based on the data reported by Jung et al. the low and high shear viscosities were assigned values of 0.056 and $0.00345 \mathrm{~Pa}$, respectively, while the time constant and the power law index were assigned values of $3.31 \mathrm{~s}$ and 0.357 , respectively.

\subsection{Boundary conditions}

During the CFD analyses the stented coronary lumen was assumed to be completely rigid and a no-slip condition was specified on both the luminal surface of the artery and the exposed stent struts. A zero relative pressure was then specified at the domain outlet and a fully developed Hagen-Poiseuille velocity profile was specified at the inlet. In order to simulate pulsatile flow conditions in a human coronary artery, a transient velocity profile was adopted from Murphy et al. to describe the variation of the centreline velocity in a left-anterior descending coronary artery during a cardiac cycle [22]. As shown in Fig. 6, the velocity profile has a peak value of $0.43 \mathrm{~m} / \mathrm{s}$ corresponding to a peak Reynolds number of 357 .

\subsection{Solution}

The conservation equations of mass and linear momentum were solved in ANSYS CFX using the coupled multigrid solver. As the peak Reynolds number for the adopted velocity profile was relatively low, the flow in the stented lumen was assumed to be laminar. To ensure that temporal convergence was achieved during the CFD analyses, preliminary cycle and time step convergence studies were carried out and the results from these studies are presented in Appendix A. Based on the results of these studies, three consecutive cycles were simulated and 32 time steps were considered in each cycle. A convergence criterion of $10^{-4}$ was specified for the velocity and density residuals in each of the CFD analyses.

\subsection{Variables of interest}

The haemodynamic environment in each of the NDF and RDF models was assessed in terms of the TAWSS, TAWSSG and OSI distributions predicted on the luminal surface of the artery. The wall shear stress (WSS) vector describes the flow-induced viscous stress exerted on the luminal surface and its magnitude is derived from the dot product of the viscous stress tensor and the associated surface normal vector as follows:

$$
|\mathrm{WSS}|=\left|n_{i} \cdot \tau_{i j}\right|
$$

here $\tau_{i j}$ denotes the viscous stress tensor and $n_{i}$ denotes the surface normal vector. The time-averaged magnitude of the WSS vector is then calculated as follows:

$$
\text { TAWSS }=\frac{1}{T} \int_{0}^{T}|\mathrm{WSS}| d t
$$

here $T$ denotes the period of the cardiac cycle. The spatial gradient of the WSS vector yields the wall shear stress gradient (WSSG) tensor, which describes the rate of change of the WSS vector with respect to Cartesian coordinates. When written with respect to local coordinates, where the local $m$ - and $n$-axes are orientated parallel and perpendicular to the direction of the WSS vector, respectively, the first and second diagonal components of the WSSG tensor are believed to have the greatest aggravating effect on the luminal surface of the artery [39]. As a result, the magnitude of the WSSG tensor is calculated as follows:

$$
\mid \text { WSSG } \mid=\sqrt{\frac{\partial \tau_{w m^{2}}}{\partial m}+\frac{\partial \tau_{w n}{ }^{2}}{\partial n}}
$$

here $\tau_{w m}$ and $\tau_{w n}$ denote the local $m$-and $n$-components of the WSS vector, respectively. The time-averaged magnitude of the WSSG tensor is then calculated as follows:

$$
\text { TAWSSG }=\frac{1}{T} \int_{0}^{T}|\mathrm{WSSG}| d t
$$

Finally, the OSI is a non-dimensional scalar variable that is often employed to evaluate the oscillatory nature of vascular flows and is calculated as follows:

$$
\text { OSI }=\frac{1}{2}\left(1-\frac{\left|\int_{0}^{T} \mathrm{WSS} d t\right|}{\int_{0}^{T}|\mathrm{WSS}| d t}\right)
$$

The OSI varies between 0 and 0.5 with a value of 0 observed in regions of unidirectional flow and a value of 0.5 observed in regions of fully-oscillatory flow.

To fully elucidate the haemodynamic environment predicted in each of the NDF and RDF models, the area-weighted mean $\mu_{w}$, standard deviation $\sigma_{w}$ and skewness $s_{w}$ of the TAWSS, TAWSSG and OSI distributions were also calculated. The area-weighted mean describes the central tendency of the distribution and is calculated as follows:

$$
\mu_{w}=\frac{\int_{w} \phi d w}{\int_{w} d w}=\frac{\sum_{i=1}^{N}\left(\Phi_{i} d w_{i}\right)}{\sum_{i=1}^{N} d w_{i}}
$$

here $\Phi_{i}$ denotes the TAWSS, TAWSSG or OSI value at the ith node, $w_{i}$ denotes the surface area at the $i$ th node and $N$ denotes the number of nodes located on the luminal surface. The area-weighted standard deviation and skewness are then calculated as follows:

$$
\begin{aligned}
& \sigma_{w}=\sqrt{\frac{\sum_{i=1}^{N}\left(\Phi_{i}-\mu_{w}\right)^{2} d w_{i}}{\sum_{i=1}^{N} d w_{i}}} \\
& s_{w}=\frac{\sum_{i=1}^{N}\left(\Phi_{i}-\mu_{w}\right)^{3} d w_{i}}{\sum_{i=1}^{N} d w_{i} \sigma_{w}{ }^{3}}
\end{aligned}
$$

The area-weighted standard deviation and skewness describe the variance and asymmetry of the distribution about the areaweighted mean, respectively. 

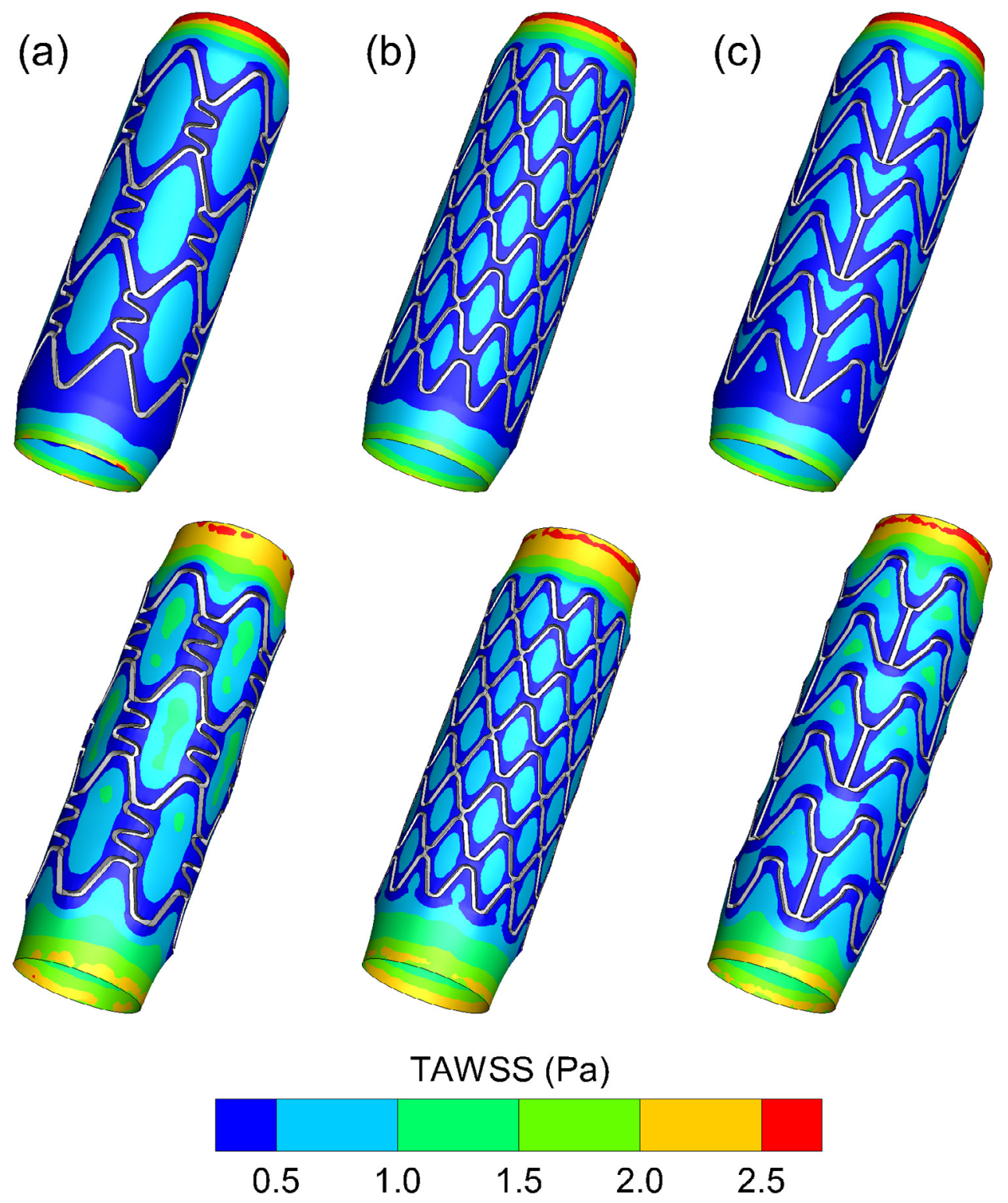

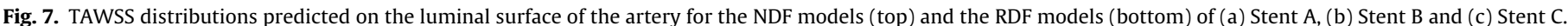
Note: the main flow direction is from the bottom to the top of the figure.

\section{Results}

\subsection{Time-averaged wall shear stress}

Contour plots of the TAWSS distributions predicted on the luminal surface for the NDF and RDF models of the investigated stents are shown in Fig. 7. When interpreting the TAWSS distributions, regions subjected to lower than physiological levels of TAWSS are believed to be at an increased risk of neointimal growth [1-4]. For both the NDF and RDF models, relatively low values of TAWSS were predicted next to the stent struts where localised regions of flow separation and recirculation were also observed. Comparing the contour plots, it is clear that stent and vessel deformation had a major impact on the TAWSS distributions predicted on the luminal surface for each of the NDF and RDF models. Specifically, a larger portion of the luminal surface was subjected to relatively low values of TAWSS in the proximal region of the NDF models. This was attributed to minor differences in the geometry of the tapered sections located at the stent extremities. For the NDF models, the geometry of the tapered sections was completely idealised and the change in diameter in these regions occurred quite gradually. For the RDF models however, the geometry of the tapered sections was dictated by the elastic response of the artery in the structural analyses. As a result, the change in diameter in these regions occurred much more abruptly.

The area-weighted mean, standard deviation and skewness of the TAWSS distribution were calculated for each of the NDF and RDF models and are given in Table 2. As shown in Table 2, the inclusion of stent and vessel deformation in the CFD analyses resulted in a $32 \%, 30 \%$ and $31 \%$ increase in the mean TAWSS for Stents A, B and C, respectively. This increase in the mean TAWSS implies that a smaller portion of the luminal surface was subjected to relatively low values of TAWSS for the RDF models. The standard deviation of the TAWSS distribution was also 15\%, 25\% and 9\% higher for the RDF models of Stents A, B and C, respectively. This increase in the standard deviation indicates a greater variance about the mean TAWSS for the RDF models. Finally, the skewness 

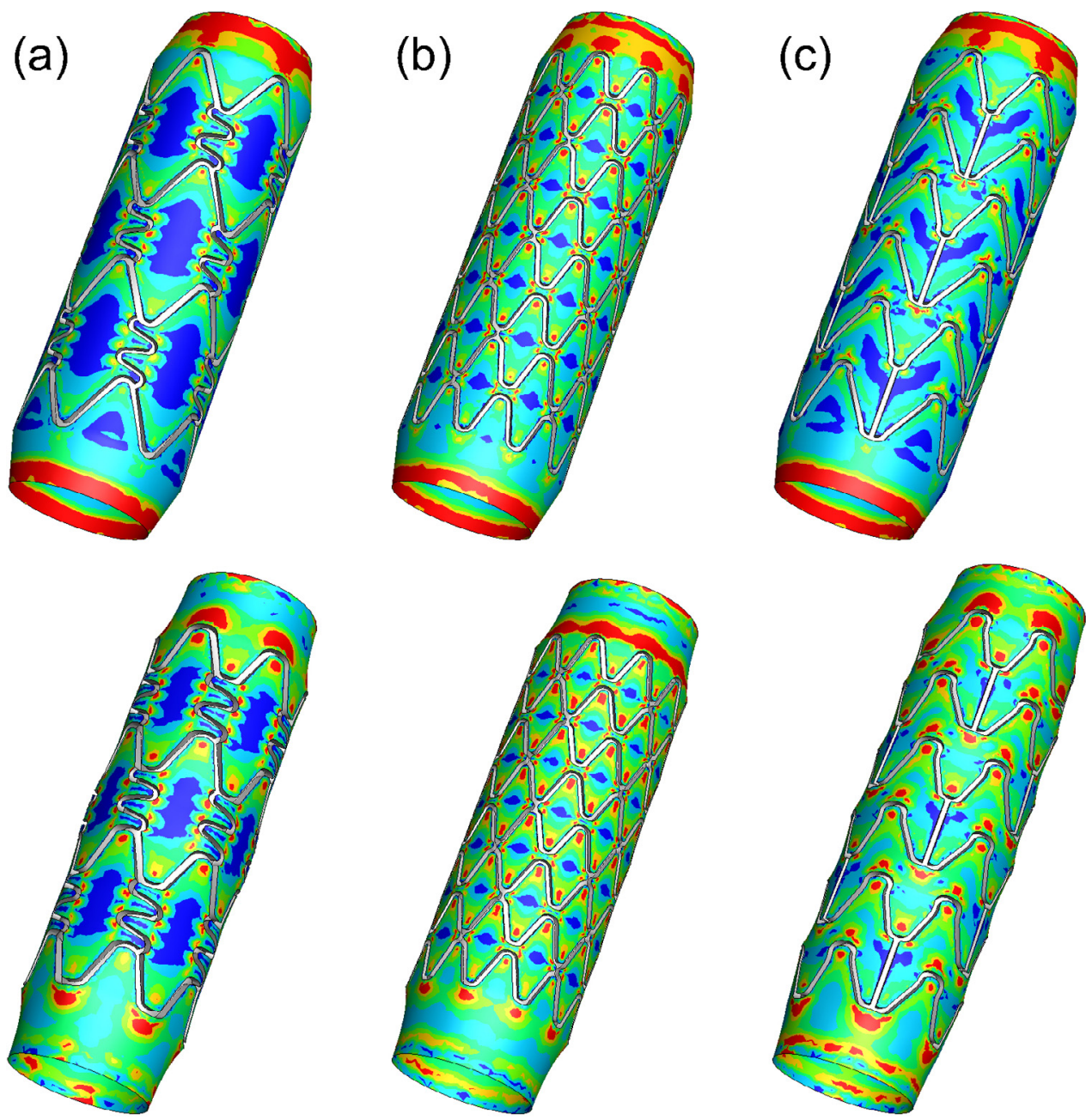

TAWSSG (Pa/mm)

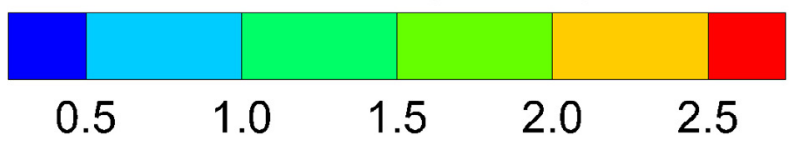

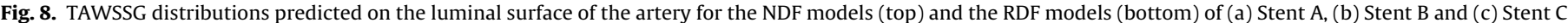
Note: the main flow direction is from the bottom to the top of the figure.

of the TAWSS distribution was $63 \%, 42 \%$ and $54 \%$ lower for the RDF models of Stents A, B and C, respectively. This decrease in the skewness implies that a lower portion of the TAWSS distribution was skewed below the mean TAWSS for the RDF models.

\subsection{Time-averaged wall shear stress gradient}

Contour plots of the TAWSSG distributions predicted on the luminal surface for the NDF and RDF models of the investigated stents are shown in Fig. 8. When interpreting the TAWSSG distributions, regions subjected to higher than physiological levels of TAWSSG are believed to be at an increased risk of neointimal growth [3]. For both the NDF and RDF models, relatively high values of TAWSSG were predicted next to the stent struts, particularly where the struts were aligned perpendicular to the main flow direction. Comparing the contour plots, it is clear that stent and vessel deformation had a major impact on the TAWSSG distributions predicted on the luminal surface for each of the NDF and RDF models.

Table 2

Area-weighted mean, standard deviation and skewness of the TAWSS distribution predicted on the luminal surface of the artery for both the NDF and RDF models of the investigated stents.

\begin{tabular}{|c|c|c|c|c|c|c|c|c|c|}
\hline \multirow[b]{2}{*}{ Model } & \multicolumn{3}{|c|}{ Stent A } & \multicolumn{3}{|c|}{ Stent B } & \multicolumn{3}{|c|}{ Stent C } \\
\hline & NDF & RDF & DIF & NDF & RDF & DIF & NDF & $\mathrm{RDF}$ & DIF \\
\hline$\mu_{w}(\mathrm{~Pa})$ & 0.638 & 0.844 & $+32 \%$ & 0.613 & 0.796 & $+30 \%$ & 0.595 & 0.781 & $+31 \%$ \\
\hline$\sigma_{w}(\mathrm{~Pa})$ & 0.557 & 0.638 & $+15 \%$ & 0.527 & 0.658 & $+25 \%$ & 0.542 & 0.592 & $+9 \%$ \\
\hline$s_{w}(-)$ & 2.264 & 0.846 & $-63 \%$ & 2.141 & 1.244 & $-42 \%$ & 2.704 & 1.248 & $-54 \%$ \\
\hline
\end{tabular}


Table 3

Area-weighted mean, standard deviation and skewness of the TAWSSG distribution predicted on the luminal surface of the artery for both the NDF and RDF models of the investigated stents.

\begin{tabular}{|c|c|c|c|c|c|c|c|c|c|}
\hline \multirow[b]{2}{*}{ Model } & \multicolumn{3}{|c|}{ Stent A } & \multicolumn{3}{|c|}{ Stent B } & \multicolumn{3}{|c|}{ Stent C } \\
\hline & NDF & RDF & DIF & NDF & RDF & DIF & NDF & RDF & DIF \\
\hline$\mu_{w}(\mathrm{~Pa} / \mathrm{mm})$ & 1.160 & 1.194 & $+3 \%$ & 1.389 & 1.491 & $+7 \%$ & 1.219 & 1.414 & $+16 \%$ \\
\hline$\sigma_{w}(\mathrm{~Pa} / \mathrm{mm})$ & 1.008 & 0.796 & $-21 \%$ & 0.831 & 0.802 & $-3 \%$ & 0.923 & 0.759 & $-18 \%$ \\
\hline$s_{w}(-)$ & 2.197 & 1.442 & $-34 \%$ & 1.123 & 1.068 & $-5 \%$ & 1.962 & 1.282 & $-35 \%$ \\
\hline
\end{tabular}

Specifically, a larger portion of the luminal surface was subjected to relatively high values of TAWSSG in the stented region of the RDF models. This was attributed to minor differences in the geometry of the stent crowns (i.e. the curved strut sections that act as hinges during stent deployment). For the NDF models, the configuration of the stent was completely idealised and the crowns followed a uniform radius of curvature. For the RDF models however, the configuration of the stent was dictated by its elastic-plastic response during the structural analyses and each of the crowns were mildly elongated. As the majority of the crowns were also aligned perpendicular to the main flow direction, this mild elongation resulted in a slight increase in the TAWSSG values observed in the stented region of the RDF models. Beyond the stented region, a greater portion of the luminal surface was subjected to relatively high values of TAWSSG in the proximal and distal regions of the NDF models. As before, this increase in the TAWSSG values was attributed to the
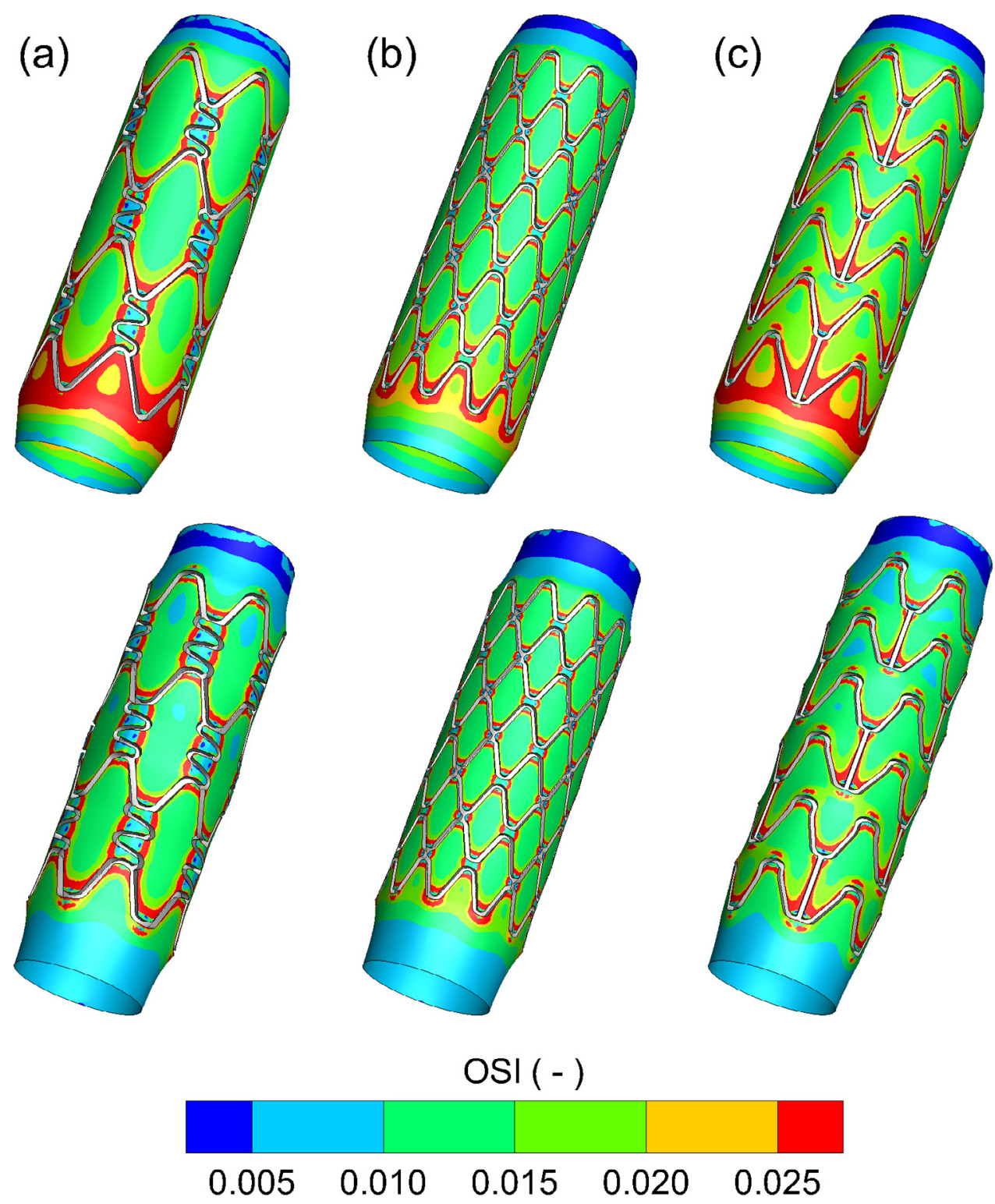

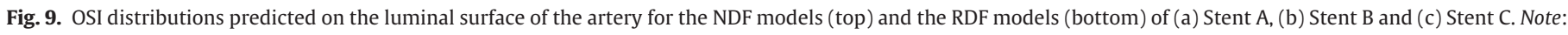
the main flow direction is from the bottom to the top of the figure.

Please cite this article in press as: Martin DM, et al. Computational fluid dynamics analysis of balloon-expandable coronary stents: Influence of stent and vessel deformation. Med Eng Phys (2014), http://dx.doi.org/10.1016/j.medengphy.2014.05.011 
Table 4

Area-weighted mean, standard deviation and skewness of the OSI distribution predicted on the luminal surface of the artery for both the NDF and RDF models of the investigated stents.

\begin{tabular}{|c|c|c|c|c|c|c|c|c|c|}
\hline \multirow[b]{2}{*}{ Model } & \multicolumn{3}{|c|}{ Stent A } & \multicolumn{3}{|c|}{ Stent B } & \multicolumn{3}{|c|}{ Stent C } \\
\hline & NDF & RDF & DIF & NDF & RDF & DIF & NDF & RDF & DIF \\
\hline$\mu_{w}(-)$ & 0.019 & 0.015 & $-21 \%$ & 0.016 & 0.014 & $-13 \%$ & 0.019 & 0.015 & $-21 \%$ \\
\hline$\sigma_{w}(-)$ & 0.020 & 0.018 & $-10 \%$ & 0.014 & 0.013 & $-7 \%$ & 0.018 & 0.017 & $-6 \%$ \\
\hline$s_{w}(-)$ & 7.36 & 9.02 & $+23 \%$ & 9.80 & 10.99 & $+12 \%$ & 9.12 & 10.15 & $+11 \%$ \\
\hline
\end{tabular}

minor differences in the geometry of the tapered sections located at the stent extremities.

The area-weighted mean, standard deviation and skewness of the TAWSSG distribution were calculated for each of the NDF and RDF models and are given in Table 3. The inclusion of stent and vessel deformation in the CFD analyses led to a 3\%, 7\% and $16 \%$ increase in the mean TAWSSG for Stents A, B and C, respectively. This increase in the mean TAWSSG implies that a larger portion of the luminal surface was subjected to relatively high values of TAWSSG for the RDF models. The standard deviation of the TAWSSG distribution was also $21 \%, 3 \%$ and $18 \%$ lower for the RDF models of Stents A, B and C, respectively. This decrease in the standard deviation indicates a lower variance about the mean TAWSSG for the RDF models. Finally, the skewness of the TAWSSG distribution was $34 \%, 5 \%$ and $35 \%$ lower for the RDF models of Stents A, B and $\mathrm{C}$, respectively. This decrease in the skewness implies that a lower portion of the TAWSSG distribution was skewed below the mean TAWSSG for the RDF models.

\subsection{Oscillatory shear index}

Contour plots of the OSI distributions predicted on the luminal surface for the NDF and RDF models of the investigated stents are shown in Fig. 9. When interpreting the OSI distributions, regions subjected to higher than physiological levels of OSI are believed to be at an increased risk of neointimal growth [40]. As the bulk flow was fully unidirectional however, the vast majority of the luminal surface was subjected to extremely low levels of OSI in each of the CFD analyses. For both the NDF and RDF models, relatively high values of OSI were predicted next to the stent struts, particularly in regions where the struts were aligned perpendicular to the main flow direction. Comparing the contour plots, it is clear that stent and vessel deformation had a major impact on the OSI distributions predicted on the luminal surface for each of the NDF and RDF models. Specifically, a larger portion of the luminal surface was subjected to relatively high values of OSI in the proximal region of the NDF models. Again, this increase in the OSI values was attributed to the minor differences in the geometry of the tapered sections located at the stent extremities.

The area-weighted mean, standard deviation and skewness of the OSI distribution were calculated for each of the NDF and RDF models and are given in Table 4 . The inclusion of stent and vessel deformation in the CFD analyses led to a $21 \%, 13 \%$ and $21 \%$ decrease in the mean OSI for Stents A, B and C, respectively. This decrease in the mean OSI implies that a smaller portion of the luminal surface was subjected to relatively high values of OSI for the RDF models. The standard deviation of the OSI distribution was also $10 \%, 7 \%$ and $6 \%$ lower for the RDF models of Stents A, B and C, respectively. This decrease in the standard deviation indicates a lower variance about the mean OSI for the RDF models. Finally, the skewness of the OSI distribution was $23 \%, 12 \%$ and $11 \%$ higher for the RDF models of Stents A, B and C, respectively. This increase in the skewness implies that a larger portion of the OSI distribution was skewed below the mean OSI for the RDF models.

\section{Discussion}

The inclusion of stent and vessel deformation in the CFD analyses led to a major increase (30-32\%) in the mean TAWSS, a moderate increase (3-16\%) in the mean TAWSSG and a moderate decrease (13-21\%) in the mean OSI for each of the investigated stents. The major increase in the mean TAWSS and the moderate decrease in the mean OSI imply that idealised NDF models of stented coronary arteries are likely to overestimate the risk of neointimal growth due to low and oscillatory WSS, respectively. Conversely, the moderate increase in the mean TAWSSG implies that idealised NDF models of stented coronary arteries are likely to underestimate the risk of neointimal growth due to elevated WSS gradients. What these results imply for in-stent restenosis is difficult to say, as it is not yet known to what extent neointimal growth is driven by low WSS, elevated WSS gradients or oscillatory WSS following coronary stent deployment. What is clear from the results is that stent and vessel deformation have a major impact on the haemodynamic environment in stented coronary arteries. Comparing the results obtained for each of the NDF and RDF models, it is also clear that the differences observed in the TAWSS, TAWSSG and OSI distributions are primarily attributed to minor differences in the geometry of the tapered sections located at the stent extremities. This highlights an important limitation of the idealised NDF approach, which requires that the configuration of these tapered sections is specified arbitrarily. It is also notable that these features (and the subsequent over-dilation of the stent) are often neglected in the literature $[6,8,11,13-15,17-21,24-28]$. In the few studies that have included idealised tapered sections with NDF models of stented coronary arteries, similar regions of low WSS were predicted in the proximal region of the artery to those observed with the NDF models in this study $[7,9,10,12]$. These results suggest that idealised NDF models are unable to provide an accurate description of the haemodynamic environment in stented coronary arteries. In light of this observation, it is recommended that realistic RDF models of stented coronary arteries are employed in future CFD studies of stented vessel haemodynamics.

Having recommended the use of RDF models in future CFD studies of stented vessel haemodynamics, it is important to note the significant computational cost associated with this approach. This computational cost is likely the main reason why idealised NDF models are generally preferred to realistic RDF models throughout the literature. In this study, the investigated stents were each assigned a uniform length of $8 \mathrm{~mm}$ as this corresponds to the shortest available length for many commercial coronary stents. Despite this simplification, the structural analyses described in this study required almost $12 \mathrm{~h}$ to complete when carried out in parallel across four compute nodes of an SGI Altix ICE 8200 EX high-performance computing (HPC) cluster, where each node featured two Xeon E5650 hex-core processors and 24GB of RAM. For comparison, the CFD analyses described in this study required almost $12 \mathrm{~h}$ to complete when carried out using a single core of a Hewlett-Packard xw8600 workstation that featured a Xeon X5450 quad-core processor and $16 \mathrm{~GB}$ of RAM. In CFD studies of stented vessel haemodynamics where much longer stents and more 
Table 5

Ranking of both the NDF and RDF models of the investigated stents from best (top) to worst (bottom) in terms of the area-weighted mean values of the TAWSS, TAWSSG and OSI distributions.

\begin{tabular}{|c|c|c|c|c|c|}
\hline \multicolumn{2}{|c|}{ TAWSS (Pa) } & \multicolumn{2}{|c|}{ TAWSSG $(\mathrm{Pa} / \mathrm{mm})$} & \multicolumn{2}{|c|}{ OSI (-) } \\
\hline NDF & $\mathrm{RDF}$ & NDF & RDF & NDF & RDF \\
\hline$A-0.638$ & $A-0.844$ & $A-1.160$ & A -1.194 & B - 0.016 & B -0.014 \\
\hline B -0.613 & B - 0.796 & $C-1.219$ & $C-1.414$ & $C-0.019$ & $C-0.015$ \\
\hline$C-0.595$ & $C-0.781$ & B - 1.389 & B - 1.491 & $A-0.019$ & $A-0.015$ \\
\hline
\end{tabular}

complex arteries are considered, the computational cost associated with the structural analyses is likely to prove unfeasible if access to a HPC cluster is not available. Although this may limit the general applicability of the RDF approach, many universities and both national and international agencies currently provide free access to powerful HPC clusters for researchers undertaking computationally expensive numerical studies. It should also be noted that although NDF models may not provide an accurate description of the haemodynamic environment in stented coronary arteries, they do appear to predict the relative performance of different stents. As shown in Table 5, the NDF and RDF models of the three investigated stents adopt identical positions when ranked from best to worst in terms of the variables of interest. This implies that the idealised NDF approach may prove an inexpensive alternative to the RDF approach in CFD studies of stented vessel haemodynamics where the relative performance of the investigated stents is the primary focus.

The results presented in this study should be interpreted in terms of several potential limitations. Firstly, the coronary artery was modelled as a straight cylindrical vessel and the presence of the atherosclerotic plaque was neglected. Though limiting, the use of a straight cylindrical artery is common throughout the literature and the effort required to simulate the deployment of the investigated stents in a realistic patient-specific model of an atherosclerotic coronary artery was deemed beyond the scope of this work. It should also be noted that idealised NDF models are unlikely to characterise the haemodynamic environment in complex coronary arteries (i.e. arteries that feature excessive deformations due to the presence of plaques, curvature, bifurcations and/or multiple stents) if they are unable to provide an accurate description of the haemodynamic environment in a simple cylindrical coronary artery. Another limitation arises from the fact that, although the structural analyses predicted regions of strut malapposition for each of the investigated stents, the struts in these regions were later extended to the luminal surface to simplify the discretisation of the RDF models. Though limiting, the idealised cylindrical geometry of the coronary artery ensured that regions of strut malapposition were both infrequent and inconsiderable. A third limitation arises from the specification of a rigid domain during the CFD analyses. This assumption is plausible however, as the deployment of a stent is likely to have a considerable stiffening effect on the lumen in the region of interest. Furthermore, a recent study carried out to investigate the impact of wall compliance in CFD studies of stented vessel haemodynamics demonstrated negligible differences in the WSS distributions obtained when both rigid and compliant domains were considered [41]. Finally, the specification of a zero relative pressure at the domain outlet results in the development of non-physiological pressure gradients during the CFD analyses. This assumption is also plausible, as the domain is assumed to be rigid and the velocity field is not influenced by the absolute values of pressure.

\section{Conclusion}

In this study, the influence of stent and vessel deformation on the haemodynamic environment in stented coronary arteries has been investigated for the first time. Comparing the results obtained with both the NDF and RDF models of the three investigated stents, it is clear that stent and vessel deformation have a major impact on the haemodynamic environment in stented coronary arteries. This impact is also clear when the area-weighted mean, standard deviation and skewness of the TAWSS, TAWSSG and OSI distributions are contrasted. Comparing the results obtained for both the NDF and RDF models, it is clear that the differences observed in the TAWSS, TAWSSG and OSI distributions are primarily attributed to minor differences in the geometry of the tapered sections located at the stent extremities. This highlights an important limitation of the idealised NDF approach, which requires that the configuration of these tapered sections is specified arbitrarily, and suggests that idealised NDF models are unable to provide an accurate description of the haemodynamic environment in simple cylindrical coronary arteries. In light of this observation, it is recommended that RDF models of stented coronary arteries are adopted in future CFD studies of stented vessel haemodynamics. This observation should aid future research in this area.

\section{Funding}

The authors would like to acknowledge funding provided by Dublin Institute of Technology under the Fiosraigh scholarship programme.

\section{Ethical approval}

No ethical approval was required for this research.

\section{Acknowledgements}

The authors would like to thank the SFI/HEA Irish Centre for High-End Computing for the provision of computational facilities and support.

\section{Appendix A. Supplementary data}

Supplementary data associated with this article can be found, in the online version, at http://dx.doi.org/10.1016/j.medengphy. 2014.05.011.

\section{Conflict of interests}

The authors have no competing interests to declare.

\section{References}

[1] Wentzel JJ, Krams R, Schuurbiers JC, Oomen JA, Kloet J, van Der Giessen WJ, et al. Relationship between neointimal thickness and shear stress after Wallstent implantation in human coronary arteries. Circulation 2001;103:1740-5.

[2] SanMartín M, Goicolea J, García C, García J, Crespo A, Rodríguez J, et al. Influence of shear stress on in-stent restenosis: in vivo study using 3D reconstruction and computational fluid dynamics. Rev Española Cardiol 2006;59:20-7.

[3] LaDisa JF, Olson LE, Molthen RC, Hettrick DA, Pratt PF, Hardel MD, et al Alterations in wall shear stress predict sites of neointimal hyperplasia after stent implantation in rabbit iliac arteries. Am J Physiol Heart Circ Physiol 2005;288:H2465-75.

[4] Carlier SG, van Damme LCA, Blommerde CP, Wentzel JJ, van Langehove G, Verheye $\mathrm{S}$, et al. Augmentation of wall shear stress inhibits neointimal hyperplasia after stent implantation: inhibition through reduction of inflammation? Circulation 2003;107:2741-6.

[5] Wentzel JJ, Whelan DM, van der Giessen WJ, van Beusekom HM, Andhyiswara I, Serruys PW, et al. Coronary stent implantation changes 3D vessel geometry and 3D shear stress distribution. J Biomech 2000;33:1287-95.

[6] LaDisa JF, Guler I, Olson LE, Hettrick DA, Kersten JR, Warltier DC, et al. Three-dimensional computational fluid dynamics modeling of alterations in coronary wall shear stress produced by stent implantation. Ann Biomed Eng 2003;31:972-80

[7] LaDisa JF, Olson LE, Guler I, Hettrick DA, Audi SH, Kersten JR, et al. Stent design properties and deployment ratio influence indexes of wall shear stress: 
a three-dimensional computational fluid dynamics investigation within a normal artery. J Appl Physiol 2004;97:424-30.

[8] He Y, Duraiswamy N, Frank AO, Moore JE. Blood flow in stented arteries: a parametric comparison of strut design patterns in three dimensions. J Biomech Eng 2005; 127:637-47.

[9] LaDisa JF, Olson LE, Guler I, Hettrick DA, Kersten JR, Warltier DC, et al. Circumferential vascular deformation after stent implantation alters wall shear stress evaluated with time-dependent 3D computational fluid dynamics models. J Appl Physiol 2005;98:947-57.

[10] LaDisa JF, Olson LE, Hettrick DA, Warltier DC, Kersten JR, Pagel PS. Axial stent strut angle influences wall shear stress after stent implantation: analysis using 3D computational fluid dynamics models of stent foreshortening. Biomed Eng Online 2005;4:59.

[11] Seo T, Schachter LG, Barakat AI. Computational study of fluid mechanical disturbance induced by endovascular stents. Ann Biomed Eng 2005;33:444-56.

[12] LaDisa JF, Olson LE, Douglas HA, Warltier DC, Kersten JR, Pagel PS. Alterations in regional vascular geometry produced by theoretical stent implantation influence distributions of wall shear stress: analysis of a curved coronary artery using 3D computational fluid dynamics modeling. Biomed Eng Online 2006;5:40.

[13] Rajamohan D, Banerjee RK, Back LH, Ibrahim AA, Jog MA. Developing pulsatile flow in a deployed coronary stent. J Biomech Eng 2006;128:347-59.

[14] Banerjee RK, Devarakonda SB, Rajamohan D, Back LH. Developed pulsatile flow in a deployed coronary stent. Biorheology 2007;44:91-102.

[15] Faik I, Mongrain R, Leask RL, Rodes-Cabau J, Larose E, Bertrand O. Timedependent 3D simulations of the hemodynamics in a stented coronary artery. Biomed Mater 2007;2:S28-37.

[16] Balossino R, Gervaso F, Migliavacca F, Dubini G. Effects of different stent designs on local hemodynamics in stented arteries. J Bomechanics 2008;41:1053-61.

[17] Dehlaghi V, Najarian S, Shadpour MT. Effect of stent geometry on phase shift between pressure and flow waveforms in stented human coronary artery. Am J Appl Sci 2008;5:340-6.

[18] Dehlaghi V, Shadpoor MT, Najarian S. Analysis of wall shear stress in stented coronary artery using 3D computational fluid dynamics modeling. J Mater Process Technol 2008;197:174-81.

[19] Chen HY, Hermiller J, Sinha AK, Sturek M, Zhu L, Kassab GS. Effects of stent sizing on endothelial and vessel wall stress: potential mechanisms for in-stent restenosis. J Appl Physiol 2009;106:1686-91.

[20] Duraiswamy N, Schoephoerster RT, Moore JE. Comparison of near-wall hemodynamic parameters in stented artery models. J Biomech Eng 2009;131:061006.

[21] Mejia J, Ruzzeh B, Mongrain R, Leask R, Bertrand OF. Evaluation of the effect of stent strut profile on shear stress distribution using statistical moments. Biomed Eng Online 2009;8:8.

[22] Murphy JB, Boyle FJ. A numerical methodology to fully elucidate the altered wall shear stress in a stented coronary artery. Cardiovasc Eng Technol 2010;1:256-68.

[23] Murphy JB, Boyle FJ. A full-range, multi-variable, CFD-based methodology to identify abnormal near-wall hemodynamics in a stented coronary artery. Biorheology 2010;47:117-32

[24] Pant S, Bressloff NW, Forrester AIJ, Curzen N. The influence of strut-connectors in stented vessels: a comparison of pulsatile flow through five coronary stents. Ann Biomed Eng 2010;38:1893-907.
[25] Williams AR, Koo B-K, Gundert TJ, Fitzgerald PJ, LaDisa JF. Local hemodynamic changes caused by main branch stent implantation and subsequent virtual side branch balloon angioplasty in a representative coronary bifurcation. J Appl Physiol 2010;109:532-40.

[26] Ellwein LM, Otake H, Gundert TJ, Koo B-K, Shinke T, Honda Y, et al. Optical coherence tomography for patient-specific 3D artery reconstruction and evaluation of wall shear stress in a left circumflex coronary artery. Cardiovasc Eng Technol 2011;2:212-27.

[27] Gundert TJ, Shadden SC, Williams AR, Koo B-K, Feinstein JA, Ladisa JF. A rapid and computationally inexpensive method to virtually implant current and next-generation stents into subject-specific computational fluid dynamics models. Ann Biomed Eng 2011;39:1423-37.

[28] Chen HY, Moussa ID, Davidson C, Kassab GS. Impact of main branch stenting on endothelial shear stress: role of side branch diameter, angle and lesion. J R Soc Interface 2012;9:1187-93.

[29] Chiastra C, Morlacchi S, Pereira S, Dubini G, Migliavacca F. Computational fluid dynamics of stented coronary bifurcations studied with a hybrid discretization method. Eur J Mech - B/Fluids 2012;35:76-84.

[30] Gundert TJ, Marsden AL, Yang W, LaDisa JF. Optimization of cardiovascular stent design using computational fluid dynamics. J Biomech Eng 2012;134:011002.

[31] Chiastra C, Morlacchi S, Gallo D, Morbiducci U, Cárdenes R, Larrabide I, et al. Computational fluid dynamic simulations of image-based stented coronary bifurcation models. J R Soc Interface 2013;10:20130193.

[32] Rikhtegar F, Pacheco F, Wyss C, Stok KS, Ge H, Choo RJ, et al. Compound ex vivo and in silico method for hemodynamic analysis of stented arteries. PLoS One 2013;8:e58147.

[33] Rikhtegar F, Wyss C, Stok KS, Poulikakos D, Müller R, Kurtcuoglu V Hemodynamics in coronary arteries with overlapping stents. J Biomech 2014:47:505-11.

[34] Martin D, Boyle F. Finite element analysis of balloon-expandable coronary stent deployment: influence of angioplasty balloon configuration. Int J Numer Method Biomed Eng 2013;29:1161-75.

[35] Murphy BP, Savage P, McHugh PE, Quinn DF. The stress-strain behavior of coronary stent struts is size dependent. Ann Biomed Eng 2003;31:686-91.

[36] Mortier P, Holzapfel GA, De Beule M, Van Loo D, Taeymans Y, Segers P, et al. A novel simulation strategy for stent insertion and deployment in curved coronary bifurcations: comparison of three drug-eluting stents. Ann Biomed Eng 2010;38:88-99.

[37] Holzapfel GA, Sommer G, Gasser CT, Regitnig P. Determination of layer-specific mechanical properties of human coronary arteries with nonatherosclerotic intimal thickening and related constitutive modeling. Am J Physiol Heart Circ Physiol 2005;289:H2048-58.

[38] Jung H, Choi JW, Park CG. Asymmetric flows of non-Newtonian fluids in symmetric stenosed artery. Korea-Australia Rheol J 2004;16:101-8.

[39] Lei M, Kleinstreuer C, Truskey GA. A focal stress gradient-dependent mass transfer mechanism for atherogenesis in branching arteries. Med Eng Phys 1996;18:326-32.

[40] Thury A. Focal in-stent restenosis near step-up: roles of low and oscillating shear stress? Circulation 2002;105:185e-7e.

[41] Chiastra C, Migliavacca F, Martínez MÁ, Malvè M. On the necessity of modelling fluid-structure interaction for stented coronary arteries. J Mech Behav Biomed Mater 2014;34:217-30. 